A N N A LES

UNIVERSITATIS MARIAE CURIE-SKŁODOWSKA

LUBLIN - POLONIA

\title{
On branchwise commutative pseudo-BCH algebras
}

\begin{abstract}
Basic properties of branches of pseudo-BCH algebras are described. Next, the concept of a branchwise commutative pseudo-BCH algebra is introduced. Some conditions equivalent to branchwise commutativity are given. It is proved that every branchwise commutative pseudo- $\mathrm{BCH}$ algebra is a pseudo-BCI algebra.
\end{abstract}

1. Introduction. In 1966, Imai and Iséki $([9,13])$ introduced BCK and $\mathrm{BCI}$ algebras. In 1983, $\mathrm{Hu}$ and $\mathrm{Li}([8])$ defined $\mathrm{BCH}$ algebras. It is known that $\mathrm{BCK}$ and $\mathrm{BCI}$ algebras are contained in the class of $\mathrm{BCH}$ algebras. In $[11,12]$, Iorgulescu introduced many interesting generalizations of BCI and BCK algebras (see also [10]).

In 2001, Georgescu and Iorgulescu ([7]) defined pseudo-BCK algebras as an extension of BCK algebras. In 2008, Dudek and Jun ([1]) introduced pseudo-BCI algebras as a natural generalization of BCI algebras and of pseudo-BCK algebras. These algebras have also connections with other algebras of logic such as pseudo-MV algebras and pseudo-BL algebras defined by Georgescu and Iorgulescu in [5] and [6], respectively. Recently, Walendziak ([14]) introduced pseudo-BCH algebras as an extension of $\mathrm{BCH}$ algebras. In $[15,16]$, he studied ideals in such algebras.

2010 Mathematics Subject Classification. 03G25, 06F35.

Key words and phrases. (Pseudo-)BCK/BCI/BCH-algebra, atom, branch, branchwise commutativity. 
In this paper we consider branches of pseudo-BCH algebras and introduce the concept of a branchwise commutative pseudo- $\mathrm{BCH}$ algebra. We show that every such algebra is a pseudo-BCI algebra. We also give some conditions equivalent to branchwise commutativity. Finally, we obtain a system of identities defining the class of branchwise commutative pseudo$\mathrm{BCH}$ algebras.

2. Preliminaries. We recall that an algebra $\mathfrak{X}=(X ; *, 0)$ of type $(2,0)$ is called a $B C H$ algebra if it satisfies the following axioms:

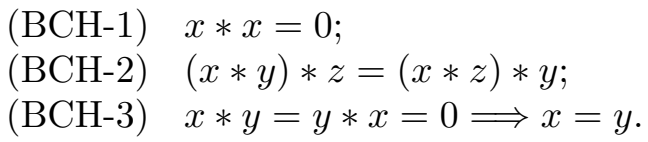

A BCH algebra $\mathfrak{X}$ is said to be a BCI algebra if it satisfies the identity

(BCI) $\quad((x * y) *(x * z)) *(z * y)=0$.

A $B C K$ algebra is a BCI algebra $\mathfrak{X}$ satisfying the law $0 * x=0$.

Definition 2.1 ([1]). A pseudo-BCI algebra is a structure $\mathfrak{X}=(X ; \leqslant, *, \diamond, 0)$, where " $\leqslant$ " is a binary relation on the set $X$, " $*$ " and " $\diamond$ " are binary operations on $X$ and " 0 " is an element of $X$, satisfying the axioms:

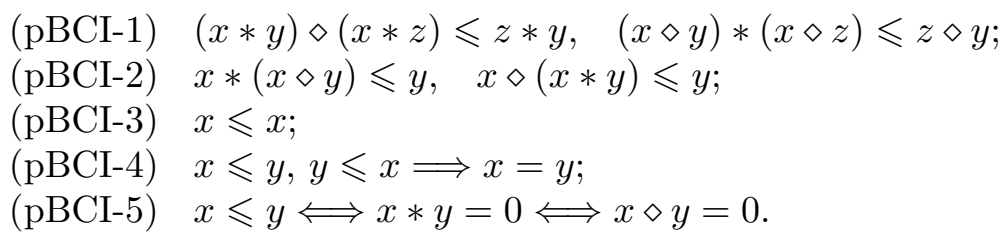

A pseudo-BCI algebra $\mathfrak{X}$ is called a pseudo-BCK algebra if it satisfies the identities

$(\mathrm{pBCK}) \quad 0 * x=0 \diamond x=0$.

Definition $2.2([14])$. A pseudo- $B C H$ algebra is an algebra $\mathfrak{X}=(X ; *, \diamond, 0)$ of type $(2,2,0)$ satisfying the axioms:

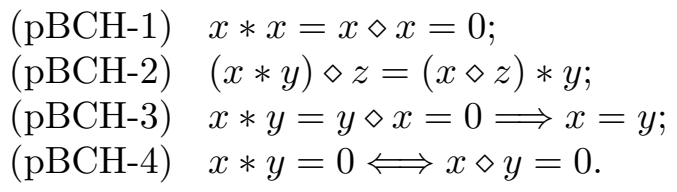

We define a binary relation $\leqslant$ on $X$ by

$$
x \leqslant y \Longleftrightarrow x * y=0 \Longleftrightarrow x \diamond y=0 .
$$

Throughout this paper $\mathfrak{X}$ will denote a pseudo-BCH algebra. 
Example 2.3 ([14], Example 4.12). Let $X=\{0, a, b, c, d\}$. Define binary operations $*$ and $\diamond$ on $X$ by the following tables:

\begin{tabular}{l|lllll}
$*$ & 0 & $a$ & $b$ & $c$ & $d$ \\
\hline 0 & 0 & 0 & 0 & 0 & $d$ \\
$a$ & $a$ & 0 & $a$ & 0 & $d$ \\
$b$ & $b$ & $b$ & 0 & 0 & $d$ \\
$c$ & $c$ & $b$ & $c$ & 0 & $d$ \\
$d$ & $d$ & $d$ & $d$ & $d$ & 0
\end{tabular}

\begin{tabular}{l|lllll}
$\diamond$ & 0 & $a$ & $b$ & $c$ & $d$ \\
\hline 0 & 0 & 0 & 0 & 0 & $d$ \\
$a$ & $a$ & 0 & $a$ & 0 & $d$ \\
$b$ & $b$ & $b$ & 0 & 0 & $d$ \\
$c$ & $c$ & $c$ & $a$ & 0 & $d$ \\
$d$ & $d$ & $d$ & $d$ & $d$ & 0
\end{tabular}

Then $\mathfrak{X}=(X ; *, \diamond, 0)$ is a pseudo-BCH algebra.

Let $\mathfrak{X}=(X ; *, \diamond, 0)$ be a pseudo-BCH algebra satisfying (pBCK), and let $(G ; \cdot, 1)$ be a group. Denote $Y=G-\{1\}$ and suppose that $X \cap Y=\emptyset$. Define the binary operations $*$ and $\diamond$ on $X \cup Y$ by

$$
x * y= \begin{cases}x * y & \text { if } x, y \in X \\ x y^{-1} & \text { if } x, y \in Y \text { and } x \neq y \\ 0 & \text { if } x, y \in Y \text { and } x=y \\ y^{-1} & \text { if } x \in X, y \in Y \\ x & \text { if } x \in Y, y \in X\end{cases}
$$

and

$$
x \diamond y= \begin{cases}x \diamond y & \text { if } x, y \in X \\ y^{-1} x & \text { if } x, y \in Y \text { and } x \neq y \\ 0 & \text { if } x, y \in Y \text { and } x=y \\ y^{-1} & \text { if } x \in X, y \in Y \\ x & \text { if } x \in Y, y \in X\end{cases}
$$

Then $(X \cup Y ; *, \diamond, 0)$ is a pseudo-BCH algebra (see [15]).

Example 2.4. Consider the set $X=\{0, a, b, c\}$ with the operation $*$ defined by the following table:

$$
\begin{array}{c|cccc}
* & 0 & a & b & c \\
\hline 0 & 0 & 0 & 0 & 0 \\
a & a & 0 & 0 & 0 \\
b & b & a & 0 & a \\
c & c & a & a & 0
\end{array}
$$

By simple calculation we can get that $\mathfrak{X}=(X ; *, 0)$ is a $\mathrm{BCH}$ algebra. Let $\mathfrak{G}$ be the group of all permutations of $\{1,2,3\}$. We have $G=\{\imath, d, e, f, g, h\}$, where $\imath=(1), d=(12), e=(13), f=(23), g=(123)$, and $h=(132)$. Applying (1) and (2), we obtain the following tables: 
A. Walendziak

\begin{tabular}{l|lllllllll}
$*$ & 0 & $a$ & $b$ & $c$ & $d$ & $e$ & $f$ & $g$ & $h$ \\
\hline 0 & 0 & 0 & 0 & 0 & $d$ & $e$ & $f$ & $h$ & $g$ \\
$a$ & $a$ & 0 & 0 & 0 & $d$ & $e$ & $f$ & $h$ & $g$ \\
$b$ & $b$ & $a$ & 0 & $a$ & $d$ & $e$ & $f$ & $h$ & $g$ \\
$c$ & $c$ & $a$ & $a$ & 0 & $d$ & $e$ & $f$ & $h$ & $g$ \\
$d$ & $d$ & $d$ & $d$ & $d$ & 0 & $h$ & $g$ & $e$ & $f$ \\
$e$ & $e$ & $e$ & $e$ & $e$ & $g$ & 0 & $h$ & $f$ & $d$ \\
$f$ & $f$ & $f$ & $f$ & $f$ & $h$ & $g$ & 0 & $d$ & $e$ \\
$g$ & $g$ & $g$ & $g$ & $g$ & $e$ & $f$ & $d$ & 0 & $h$ \\
$h$ & $h$ & $h$ & $h$ & $h$ & $f$ & $d$ & $e$ & $g$ & 0
\end{tabular}

and

\begin{tabular}{l|lllllllll}
$\diamond$ & 0 & $a$ & $b$ & $c$ & $d$ & $e$ & $f$ & $g$ & $h$ \\
\hline 0 & 0 & 0 & 0 & 0 & $d$ & $e$ & $f$ & $h$ & $g$ \\
$a$ & $a$ & 0 & 0 & 0 & $d$ & $e$ & $f$ & $h$ & $g$ \\
$b$ & $b$ & $a$ & 0 & $a$ & $d$ & $e$ & $f$ & $h$ & $g$ \\
$c$ & $c$ & $a$ & $a$ & 0 & $d$ & $e$ & $f$ & $h$ & $g$ \\
$d$ & $d$ & $d$ & $d$ & $d$ & 0 & $g$ & $h$ & $f$ & $e$ \\
$e$ & $e$ & $e$ & $e$ & $e$ & $h$ & 0 & $g$ & $d$ & $f$ \\
$f$ & $f$ & $f$ & $f$ & $f$ & $g$ & $h$ & 0 & $e$ & $d$ \\
$g$ & $g$ & $g$ & $g$ & $g$ & $f$ & $d$ & $e$ & 0 & $h$ \\
$h$ & $h$ & $h$ & $h$ & $h$ & $e$ & $f$ & $d$ & $g$ & 0
\end{tabular}

Then $(\{0, a, b, c, d, e, f, g, h\} ; *, \diamond, 0)$ is a pseudo-BCH algebra.

From [14] it follows that in any pseudo-BCH algebra $\mathfrak{X}$, for all $x, y \in X$, we have:

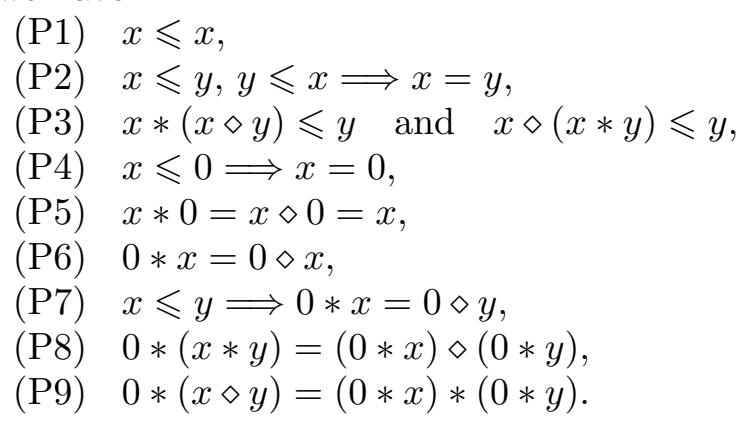

Remark. By Theorem 3.4 of [14], a pseudo-BCH algebra is a pseudo-BCI algebra if and only if it satisfies the following implication:

$$
x \leqslant y \Longrightarrow(x * z \leqslant y * z, x \diamond z \leqslant y \diamond z) .
$$

Proposition 2.5. For a pseudo- $B C H$ algebra $\mathfrak{X}$ the following conditions are equivalent:
(a) $\mathfrak{X}$ is a pseudo-BCI algebra,
(b) $\mathfrak{X}$ satisfies axiom (pBCI-1),
(c) $\mathfrak{X}$ satisfies condition $(*)$. 
Proof. The equivalence of (a) and (c) follows from the above remark.

(a) $\Longrightarrow($ b) is obvious.

(b) $\Longrightarrow(\mathrm{a})$ : By assumption, $\mathfrak{X}$ satisfies (pBCI-1) and (pBCI-5). The axioms (pBCI-2)-(pBCI-4) follow from the properties (P1)-(P3).

3. Atoms and branches. An element $a$ of $\mathfrak{X}$ is called an atom if $x \leqslant a$ implies $x=a$ for all $x \in X$, that is, $a$ is a minimal element of $(X ; \leqslant)$. Let us denote by $\mathrm{A}(\mathfrak{X})$ the set of all atoms of $\mathfrak{X}$. By $(\mathrm{P} 4), 0 \in \mathrm{A}(\mathfrak{X})$.

Proposition 3.1 ([14], Propositions 4.1 and 4.2). Let $\mathfrak{X}$ be a pseudo-BCHalgebra and let $a \in X$. Then the following conditions are equivalent:

(i) $a$ is an atom,

(ii) $x \diamond(x * a)=a$ for all $x \in X$,

(iii) $0 \diamond(0 * a)=a$,

(iv) $x *(x \diamond a)=a$ for all $x \in X$,

(v) $0 *(0 \diamond a)=a$.

Proposition 3.2 ([14], Proposition 4.3). Let $\mathfrak{X}$ be a pseudo-BCH algebra and let $a \in X$. Then $a$ is an atom if and only if there is an element $x \in X$ such that $a=0 * x$.

As a consequence of Proposition 3.2, we obtain

Corollary 3.3. For every $x \in X$, we have $0 * x \in \mathrm{A}(\mathfrak{X})$.

For $x \in X$, set

$$
\bar{x}=0 \diamond(0 * x) .
$$

By $(\mathrm{P} 6), \bar{x}=0 *(0 * x)=0 \diamond(0 \diamond x)=0 *(0 \diamond x)$. Note that the map $\varphi(x)=0 *(0 * x)$ was introduced in [17] for BZ algebras (such algebras are a generalization of BCI algebras). Different properties of this map were used in many papers (for example, [18], [2] and [3]).

Proposition 3.4 ([14], Proposition 4.4). Let $\mathfrak{X}$ be a pseudo-BCH algebra. For any $x, y \in X$ we have:

(i) $\overline{x * y}=\bar{x} * \bar{y}$,

(ii) $\overline{x \diamond y}=\bar{x} \diamond \bar{y}$,

(iii) $\overline{\bar{x}}=\bar{x}$.

For BZ algebras, (iii) was proved in [17]. In [14], the set $\{x \in X: x=\bar{x}\}$ is called the centre of $\mathfrak{X}$ and it is denoted by Cen $\mathfrak{X}$. We conclude from Proposition 3.1 that $\mathrm{Cen} \mathfrak{X}=\mathrm{A}(\mathfrak{X})$. Then $\mathrm{A}(\mathfrak{X})=\{\bar{x}: x \in X\}$. By Proposition $3.4, \mathrm{~A}(\mathfrak{X})$ is a subalgebra of $\mathfrak{X}$.

For any pseudo-BCH algebra $\mathfrak{X}$, we set

$$
\mathrm{K}(\mathfrak{X})=\{x \in X: 0 \leqslant x\} .
$$

From Corollary 4.19 of [14] it follows that $\mathrm{K}(\mathfrak{X})$ is a subalgebra of $\mathfrak{X}$. 
Observe that

$$
\mathrm{A}(\mathfrak{X}) \cap \mathrm{K}(\mathfrak{X})=\{0\} .
$$

Indeed, $0 \in \mathrm{A}(\mathfrak{X}) \cap \mathrm{K}(\mathfrak{X})$ and if $x \in \mathrm{A}(\mathfrak{X}) \cap \mathrm{K}(\mathfrak{X})$, then $x=0 *(0 * x)=$ $0 * 0=0$.

Lemma 3.5. Let $x, y \in X$. If $x * y \in \mathrm{K}(\mathfrak{X})$, then $y * x, x \diamond y, y \diamond x \in \mathrm{K}(\mathfrak{X})$.

Proof. Let $x * y \in \mathrm{K}(\mathfrak{X})$. Then $0 *(x * y)=0$. We deduce from $(\mathrm{P} 8)$ that $(0 * x) \diamond(0 * y)=0$, and hence $0 * x \leqslant 0 * y$. Since $0 * x, 0 * y \in \mathrm{A}(\mathfrak{X})$ (see Corollary 3.3), we have $0 * x=0 * y$. Consequently,

$$
0 *(y * x)=(0 * y) \diamond(0 * x)=(0 * y) \diamond(0 * y)=0,
$$

that is, $0 *(y * x)=0$. Applying $(\mathrm{P} 9)$, we also deduce that $0 *(x \diamond y)=0$ and $0 *(y \diamond x)=0$. Therefore, $y * x, x \diamond y, y \diamond x \in \mathrm{K}(\mathfrak{X})$.

For any element $a$ of a pseudo-BCH-algebra $\mathfrak{X}$, we define a subset $\mathrm{V}(a)$ of $X$ as

$$
\mathrm{V}(a)=\{x \in X: a \leq x\} .
$$

Note that $\mathrm{V}(a) \neq \emptyset$, because $a \leq a$ gives $a \in \mathrm{V}(a)$. Furthermore, $\mathrm{V}(0)=$ $\mathrm{K}(\mathfrak{X})$. If $a \in \mathrm{A}(\mathfrak{X})$, then the set $\mathrm{V}(a)$ is called a branch of $\mathfrak{X}$ determined by element $a$.

Example 3.6. Let $\mathfrak{X}=(\{0, a, b, c, d\} ; *, \diamond, 0)$ be the pseudo-BCH algebra given in Example 2.3. It is easily seen that $\mathrm{A}(\mathfrak{X})=\{0, d\}$ and $\mathfrak{X}$ has two branches $\mathrm{V}(0)=\{0, a, b, c\}$ and $\mathrm{V}(d)=\{d\}$.

Example 3.7. Let $\mathfrak{X}=(\{0, a, b, c, d, e, f, g, h\} ; *, \diamond, 0)$ be the pseudo-BCH algebra from Example 2.4. Obviously, $\mathrm{A}(\mathfrak{X})=\{0, d, e, f, g, h\}$. The algebra $\mathfrak{X}$ has the following branches: $\mathrm{V}(0)=\{0, a, b, c\}, \mathrm{V}(d)=\{d\}, \mathrm{V}(e)=\{e\}$, $\mathrm{V}(f)=\{f\}, \mathrm{V}(g)=\{g\}, \mathrm{V}(h)=\{h\}$.

Proposition 3.8 ([14], Proposition 4.23). Let $\mathfrak{X}$ be a pseudo-BCH algebra. Then:

(i) $X=\bigcup\{\mathrm{V}(a): a \in \mathrm{A}(\mathfrak{X})\}$

(ii) if $a, b \in \mathrm{A}(\mathfrak{X})$ and $a \neq b$, then $V(a) \cap \mathrm{V}(b)=\emptyset$.

Proposition 3.9. Two elements $x, y$ are in the same branch of $\mathfrak{X}$ if and only if $x * y \in \mathrm{K}(\mathfrak{X})$ (or equivalently, $x \diamond y \in \mathrm{K}(\mathfrak{X})$ ).

Proof. If $x$ and $y$ are in the same branch $\mathrm{V}(a)$, then $a \leqslant x$ and $a \leqslant y$. By (P6) and (P7), $0 * x=0 * a=0 * y$. Applying (P8), we obtain $0 *(x * y)=$ $(0 * x) \diamond(0 * y)=0$. Thus $0 \leqslant x * y$, that is, $x * y \in \mathrm{K}(\mathfrak{X})$.

Conversely, suppose that $x * y \in \mathrm{K}(\mathfrak{X})$ and $x \in \mathrm{V}(a), y \in \mathrm{V}(b)$ for some $a, b \in \mathrm{A}(\mathfrak{X})$. Hence $a \leqslant x$ and $b \leqslant y$. Using (P6) and (P7), we get $0 * a=0 * x$ and $0 * b=0 * y$. Therefore, $a=\bar{x}$ and $b=\bar{y}$. From Proposition 3.4 we have $\overline{x * y}=\bar{x} * \bar{y}=a * b$ and $\overline{y \diamond x}=b \diamond a$. Since $x * y \in \mathrm{K}(\mathfrak{X})$ and also $y \diamond x \in \mathrm{K}(\mathfrak{X})$ (see Lemma 3.5) we conclude that $\overline{x * y}=\overline{y \diamond x}=0$. Therefore, $a * b=b \diamond a=0$ which gives $a=b$. So $x$ and $y$ are in the same branch. 
Proposition 3.10. Comparable elements of $\mathfrak{X}$ are in the same branch.

Proof. Let $x, y \in X$ and let $x \leqslant y$. Then $x * y=0 \in \mathrm{K}(\mathfrak{X})$. By Proposition $3.9, x$ and $y$ are in the same branch.

Proposition 3.11. If elements $x$ and $y$ are comparable, then $x * y, y * x$, $x \diamond y, y \diamond x \in \mathrm{K}(\mathfrak{X})$.

Proof. From Propositions 3.10 and 3.9 we see that $x * y \in \mathrm{K}(\mathfrak{X})$ and hence $y * x, x \diamond y, y \diamond x \in \mathrm{K}(\mathfrak{X})$ by Lemma 3.5 .

4. Branchwise commutativity. A pseudo- $\mathrm{BCH}$ algebra $\mathfrak{X}$ is said to be commutative if for all $x, y \in X$, it satisfies the following identities:

$$
\begin{aligned}
& x *(x \diamond y)=y *(y \diamond x), \\
& x \diamond(x * y)=y \diamond(y * x) .
\end{aligned}
$$

Proposition 4.1. Every commutative pseudo- $B C H$ algebra is a pseudo$B C K$ algebra.

Proof. Let $\mathfrak{X}$ be a commutative pseudo- $\mathrm{BCH}$ algebra. First observe that $\mathfrak{X}$ satisfies (pBCK). Let $x \in X$. Applying (pBCH-1), (P5) and (P3), we obtain

$$
0=x * x=x *(x \diamond 0)=0 *(0 \diamond x) \leqslant x .
$$

Then $0 * x=0 \diamond x=0$, that is, (pBCK) holds.

Now we show that $\mathfrak{X}$ satisfies (pBCI-1). Let $x, y \in X$. We have

$$
\begin{aligned}
((x * y) \diamond(x * z)) *(z * y) & =((x \diamond(x * z)) * y) *(z * y) & & {[\text { by }(\mathrm{pBCH}-2)] } \\
& =((z \diamond(z * x)) * y) *(z * y) & & {[\text { by }(4)] } \\
& =((z * y) *(z * y)) \diamond(z * x) & & {[\text { by }(\mathrm{pBCH}-2)] } \\
& =0 \diamond(z * x) & & {[\text { by }(\mathrm{pBCH}-1)] } \\
& =0 & & {[\text { by }(\mathrm{pBCK})] }
\end{aligned}
$$

and hence $(x * y) \diamond(x * z) \leqslant(z * y)$. Similarly, $(x \diamond y) *(x \diamond z) \leqslant z \diamond y$. Thus (pBCI- 1 ) holds in $\mathfrak{X}$. We conclude from Proposition 2.5 that $\mathfrak{X}$ is a pseudo-BCI algebra, and finally that it is a pseudo-BCK algebra.

Corollary 4.2. Commutative pseudo-BCH algebras coincide with commutative pseudo-BCK algebras.

In [4], G. Dymek introduced the notion of branchwise commutative pseudo-BCI algebras. Following [4], we say that a pseudo-BCH algebra $\mathfrak{X}$ is branchwise commutative if identities (3) and (4) hold for $x$ and $y$ belonging to the same branch. Clearly, any commutative pseudo-BCH algebra is branchwise commutative. 
Remark. Note that the pseudo-BCH algebra from Example 2.4 is branchwise commutative but it is not commutative, since $d \diamond(d * a)=0 \neq d=$ $a \diamond(a * d)$.

The algebra given in Example 2.3 is not branchwise commutative. Indeed, $a *(a \diamond c)=a$ but $c *(c \diamond a)=0$.

Proposition 4.3 ([4], Theorem 3.2). A pseudo-BCI algebra $(X ; \leqslant, *, \diamond, 0)$ is branchwise commutative if and only if for all $x, y \in X$, satisfies the following condition:

$$
x \leqslant y \Longrightarrow x=y \diamond(y * x)=y *(y \diamond x) .
$$

Lemma 4.4. If $\mathfrak{X}$ satisfies $(\mathrm{BC})$, then $\mathfrak{X}$ is a pseudo-BCI algebra.

Proof. Let $x, y \in X$ and $x \leqslant y$. We have

$$
\begin{aligned}
(x * z) \diamond(y * z) & =((y \diamond(y * x)) * z) *(y * z) & & {[\text { since } x=y \diamond(y * x)] } \\
& =((y * z) \diamond(y * x)) *(y * z) & & {[\text { by }(\mathrm{pBCH}-2)] } \\
& =((y * z) *(y * z)) \diamond(y * x) & & {[\text { by }(\mathrm{pBCH}-2)] } \\
& =0 \diamond(y * x) & & {[\text { by }(\mathrm{pBCH}-1)] . }
\end{aligned}
$$

Since elements $x$ and $y$ are comparable, by Proposition 3.11, $y * x \in \mathrm{K}(\mathfrak{X})$. Therefore, $0 \diamond(y * x)=0$, and hence $(x * z) \diamond(y * z)=0$. Consequently, $x * z \leqslant y * z$. Similarly, $x \diamond z \leqslant y \diamond z$. From Proposition 2.5 it follows that $\mathfrak{X}$ is a pseudo-BCI algebra.

As a consequence of the above lemma and Proposition 4.3, we obtain:

Proposition 4.5. If a pseudo-BCH algebra satisfies (BC), then it is branchwise commutative.

Theorem 4.6. Any branchwise commutative pseudo-BCH algebra is a pseudo-BCI algebra.

Proof. Let $\mathfrak{X}$ be a brachwise commutative pseudo-BCH algebra. Let $x, y \in$ $X$ and $x \leqslant y$. Then $x * y=0$. By Proposition 3.10, elements $x$ and $y$ are in the same branch. Since $\mathfrak{X}$ is brachwise commutative, we obtain

$$
y \diamond(y * x)=x \diamond(x * y)=x \diamond 0=x .
$$

Similarly, we prove that $x=y *(y \diamond x)$. Thus condition (BC) holds in $\mathfrak{X}$. From Lemma 4.4 we conclude that $\mathfrak{X}$ is a pseudo-BCI algebra.

Corollary 4.7. Branchwise commutative pseudo- $B C H$ algebras coincide with branchwise commutative pseudo-BCI algebras.

As a consequence of Corollary 4.7, all results holding for branchwise commutative pseudo-BCI algebras also hold for brachwise commutative pseudo$\mathrm{BCH}$ algebras. We recall some of these results: 
Proposition 4.8 ([4]). Let $\mathfrak{X}$ be a branchwise commutative pseudo-BCH/ $B C I$ algebra. Then:

(i) for all $x, y \in X$, we have

$$
\begin{aligned}
& x \diamond(x * y)=y \diamond(y *(x \diamond(x * y))), \\
& x *(x \diamond y)=y *(y \diamond(x *(x \diamond y))) .
\end{aligned}
$$

(ii) for all $x$ and $y$ belonging to the same branch,

$$
\begin{aligned}
& x * y=x *(y \diamond(y * x)), \\
& x \diamond y=x \diamond(y *(y \diamond x)) .
\end{aligned}
$$

(iii) each branch of $\mathfrak{X}$ is a semilattice with respect to the operation $\wedge$ defined by $x \wedge y=y \diamond(y * x)=y *(y \diamond x)$.

Theorem 4.9. Let $\mathfrak{X}$ be a pseudo- $B C H$ algebra. The following are equivalent:

(a) $\mathfrak{X}$ is branchwise commutative,

(b) $\mathfrak{X}$ satisfies $(\mathrm{BC})$,

(c) $\mathfrak{X}$ satisfies (5) and (6),

(d) the identities (7) and (8) hold for all $x$ and $y$ belonging to the same branch of $\mathfrak{X}$,

(e) each branch of $\mathfrak{X}$ is a semilattice with respect to the operation $\wedge$ defined by $x \wedge y=y \diamond(y * x)=y *(y \diamond x)$.

Proof. Let $\mathfrak{X}$ be a branchwise commutative pseudo-BCH algebra. Then, by Theorem $4.6, \mathfrak{X}$ is a branchwise commutative pseudo-BCI algebra. From Propositions 4.3 and 4.8 we deduce that (a) implies (b), (c), (d) and (e).

(c) $\Longrightarrow(\mathrm{b})$ : Let $x, y \in X$ and $x \leqslant y$. Then $x * y=0$. From (5) we see that $x=y \diamond(y * x)$. Similarly, from (6) we get $x=y *(y \diamond x)$. Therefore, (BC) holds in $\mathfrak{X}$.

(d) $\Longrightarrow($ b): Suppose that $x \leqslant y$. By Proposition 3.10, elements $x$ and $y$ are in the same branch. Putting $x * y=0$ in (7) and $x \diamond y=0$ in (8), we get $0=x *(y \diamond(y * x))=x \diamond(y *(y \diamond x))$. Hence $x \leqslant y \diamond(y * x)$ and $x \leqslant y *(y \diamond x)$. Applying (P3), we have $y \diamond(y * x) \leqslant x$ and $y *(y \diamond x) \leqslant x$. Thus $x=y \diamond(y * x)=y *(y \diamond x)$. Consequently, $\mathfrak{X}$ satisfies (BC).

(e) $\Longrightarrow(\mathrm{b})$ : If $x \leqslant y$, then $x, y$ are in the same branch and, by (e), $x=x \wedge y=y \diamond(y * x)=y *(y \diamond x)$. Therefore, we obtain (b).

(b) $\Longrightarrow$ (a) follows from Proposition 4.5.

In [4], Dymek obtained an axiomatization of branchwise commutative pseudo-BCI algebras. We give an alternative axiomatization of such algebras. 
Theorem 4.10. An algebra $\mathfrak{X}=(X ; *, \diamond, 0)$ of type $(2,2,0)$ is a branchwise commutative pseudo-BCH algebra if and only if it satisfies the following identities:

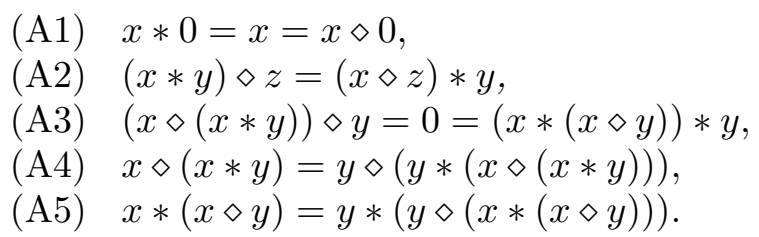

Proof. If $\mathfrak{X}$ is a branchwise commutative pseudo-BCH algebra, then, obviously, the identities (A1)-(A5) hold for all $x, y \in X$. Conversely, suppose that $\mathfrak{X}$ satisfies (A1)-(A5). Putting $y=0$ in (A3) and applying (A1), we obtain (pBCH-1). To prove (pBCH-3), let $x * y=y * x=0$. Using (A1) and (A4), we get

$$
x=x \diamond 0=x \diamond(x * y)=y \diamond(y *(x \diamond(x * y)))=y \diamond(y * x)=y \diamond 0=y,
$$

that is, (pBCH-3) holds in $\mathfrak{X}$. We now prove that

$$
x * y=0 \Longleftrightarrow x \diamond y=0 .
$$

If $x * y=0$, then $(x \diamond 0) \diamond y=0$ by (A3), and hence $x \diamond y=0$. Thus $x * y=0$ implies $x \diamond y=0$, and analogously, $x \diamond y=0$ entails $x * y=0$. Therefore $\mathfrak{X}$ satisfies (pBCH-4), and finally, it is a pseudo-BCH algebra. Moreover, $\mathfrak{X}$ is branchwise commutative by Theorem 4.9 .

Remark. From Theorem 3.11 of [4] we see that the variety of all branchwise commutative pseudo-BCH/BCI algebras is weakly regular.

\section{REFERENCES}

[1] Dudek, W. A., Jun, Y. B., Pseudo-BCI-algebras, East Asian Math. J. 24 (2008), $187-190$.

[2] Dudek, W. A., Zhang, X., Wang, Y., Ideals and atoms of BZ-algebras, Math. Slovaca 59 (2009), 387-404.

[3] Dudek, W. A., Karamdin, B., Bhatti, S. A., Branches and ideals of weak BCCalgebras, Algebra Colloquium 18 (Special) (2011), 899-914.

[4] Dymek, G., On two classes of pseudo-BCI-algebras, Discuss. Math. Gen. Algebra Appl. 31 (2011), 217-230.

[5] Georgescu, G., Iorgulescu, A., Pseudo-MV algebras: a noncommutative extension of MV algebras, in: The Proc. of the Fourth International Symp. on Economic Informatics, Bucharest, Romania, May 1999, 961-968.

[6] Georgescu, G., Iorgulescu, A., Pseudo-BL algebras: a noncommutative extension of BL algebras, in: Abstracts of the Fifth International Conference FSTA 2000, Slovakia, February 2000, 90-92.

[7] Georgescu, G., Iorgulescu, A., Pseudo-BCK algebras: an extension of BCK algebras, in: Proc. of DMTCS'01: Combinatorics, Computability and Logic, Springer, London, 2001, 97-114.

[8] Hu, Q. P., Li, X., On BCH-algebras, Math. Seminar Notes 11 (1983), 313-320.

[9] Imai, Y., Iséki, K., On axiom systems of propositional calculi XIV, Proc. Japan Acad. Ser. A Math. Sci. 42 (1966), 19-22. 
[10] Iorgulescu A., Algebras of Logic as BCK-Algebras, Bucharest 2008.

[11] Iorgulescu, A., New generalizations of BCI, BCK and Hilbert algebras - Part I, J. Mult.-Valued Logic Soft Comput. 27 (2016), 353-406.

[12] Iorgulescu, A., New generalizations of BCI, BCK and Hilbert algebras - Part II, J. Mult.-Valued Logic Soft Comput. 27 (2016), 407-456.

[13] Iséki, K., An algebra related with a propositional calculus, Proc. Japan Acad. Ser. A Math. Sci. 42 (1966), 26-29.

[14] Walendziak, A., Pseudo-BCH-algebras, Discuss. Math. Gen. Algebra Appl. 35 (2015), $1-15$.

[15] Walendziak, A., On ideals of pseudo-BCH-algebras, Ann. Univ. Mariae CurieSkłodowska Sect. A 70 (2016), 81-91.

[16] Walendziak, A., Strong ideals and horizontal ideals in pseudo-BCH-algebras, Ann. Univ. Paedagog. Crac. Stud. Math. 15 (2016), 15-25.

[17] Zhang, X., Ye, R., BZ-algebra and group, J. of Mathematical and Physical Sciences 29 (1995), 223-233.

[18] Zhang, X., Wang, Y., Dudek, W. A., T-ideals in BZ-algebras and T-type BZ-algebras, Indian J. Pure Appl. Math. 34 (2003), 1559-1570.

Andrzej Walendziak

Institute of Mathematics and Physics

Faculty of Science

Siedlce University of Natural Sciences and Humanities

3 Maja 54, PL-08110 Siedlce

Poland

e-mail: walent@interia.pl

Received December 1, 2016 